Dev Neurosci 2018;40:638-650

DOI: $10.1159 / 000499126$
Received: October 9, 2018

Accepted after revision: February 25, 2019

Published online: June 17, 2019

\title{
Perinatal Ischemia Alters Global Expression of Synaptosomal Proteins Critical for Neural Plasticity in the Developing Mouse Brain
}

\author{
Amanda K. Barks ${ }^{a}$ Montana M. Beeson ${ }^{a} \quad$ Tatyana Matveeva ${ }^{b}$ Jonathan J. Gale \\ Raghavendra Rao ${ }^{a}$ Phu V. Tran ${ }^{a}$ \\ ${ }^{a}$ Department of Pediatrics, University of Minnesota, Minneapolis, MN, USA; ${ }^{b}$ Department of Psychology, University \\ of Minnesota, Minneapolis, MN, USA
}

\section{Keywords}

Neonatal ischemia $\cdot$ Synaptosome $\cdot$ Neuronal plasticity

Proteome $\cdot$ Brain injury

\begin{abstract}
Ischemic perinatal stroke (IPS) affects 1 in 2,300-5,000 live births. Despite a survival rate $>95 \%$, approximately $60 \%$ of IPS infants develop motor and cognitive impairments. Given the importance of axonal growth and synaptic plasticity in neurocognitive development, our objective was to identify the molecular pathways underlying IPS-associated synaptic dysfunction using a mouse model. IPS was induced by unilateral ligation of the common carotid artery of postnatal day 10 (P10) mice. Five days after ischemia, sensorimotor and motor functions were assessed by vibrissae-evoked forepaw placement and the tail suspension test respectively, showing evidence of greater impairments in male pups than in female pups. Twenty-four hours after ischemia, both hemispheres were collected and synaptosomal proteins then prepared for quantification, using isobaric tags for relative and absolute quantitation. Seventy-two of 1,498 qualified proteins were altered in the ischemic hemisphere. Ingenuity Pathway Analysis was used to map these proteins onto mo-
\end{abstract}

\begin{tabular}{ll}
\hline KARGER & $\begin{array}{l}\text { (c) } 2019 \text { The Author(s) } \\
\text { Published by S. Karger AG, Basel }\end{array}$ \\
E-Mail karger@karger.com & $\begin{array}{l}\text { This article is licensed under the Creative Commons Attribution- } \\
\text { NonCommercial-NoDerivatives 4.0 International License (CC BY- } \\
\text { NC-ND) (http://www.karger.com/Services/OpenAccessLicense). } \\
\text { Usage and distribution for commercial purposes as well as any dis- } \\
\text { tribution of modified material requires written permission. }\end{array}$
\end{tabular}

lecular networks indicative of reduced neuronal proliferation, survival, and synaptic plasticity, accompanied by reduced PKCa signaling in male, but not female, pups. These effects also occurred in the non-ischemic hemisphere when compared with sham controls. The altered signaling effects may contribute to the sex-specific neurodevelopmental dysfunction following IPS, highlighting potential pathways for targeting during treatment.

(c) 2019 The Author(s) Published by S. Karger AG, Basel

\section{Introduction}

Ischemic perinatal stroke (IPS) is a significant cause of long-term neurologic and cognitive disability including cerebral palsy, epilepsy, neurodevelopmental impairments, and behavioral disorders [1,2]. IPS thus constitutes a major long-term cost to society in terms of special education and health care [3]. The incidence of perinatal stroke is high, occurring in 1 of $2,300-5,000$ live births $[2,4,5]$. The underlying mechanisms of perinatal stroke injury and repair remain poorly understood, partly due to the wide range of pathophysiology, from asymptomatic to hemorrhagic-encephalopathic 
presentation [6]. Consequently, there remains a challenge to develop targeted and effective treatment approaches for the condition. Moreover, given the sex-dependent incidence and outcomes of IPS in humans, i.e., IPS results in more neurological deficits in male than female infants [7-9], understanding the underlying molecular mechanisms of long-term effects in IPS survivors is of major interest. This can provide an insight into the specific neurodevelopmental processes that are affected by IPS and may lead to novel strategies to prevent or mitigate the adverse long-term outcomes. Despite the recognition that neonatal brain injuries, including IPS, involve complex mechanistic cascades that can be detrimental to the developing brain $[2,10,11]$, the major pathways and mechanisms underlying IPS-induced synaptic plasticity and dysfunction $[12,13]$ are poorly studied. Changes in specific synaptic proteins have previously been shown in the adult brain following ischemic stroke [14-17]. There is a report demonstrating acute changes in synaptic protein following neonatal hypoxiaischemia injury [18], but comprehensive analyses of synaptosomal proteins altered by IPS in the developing brain remain limited.

One approach to the analysis of the synaptic proteome is through quantitative proteomic analysis using the isobaric tags for relative and absolute quantitation (iTRAQ) method [19-21]. Using the iTRAQ technique, the protein content and composition in synaptosomes can be quantified in the ischemic (ipsilateral [IL] to the ligated common carotid artery) and non-ischemic (contralateral [CL] to the ligated common carotid artery) hemispheres. Subsequently, the iTRAQ data can be functionally annotated to identify molecular networks using the knowledge-based Ingenuity Pathway Analysis (IPA) database (Qiagen, Redwood City, CA, USA), which is a collection of experimental findings from biomedical literature and third-party databases.

The objective of this study was to induce IPS in postnatal day 10 (P10) mouse pups, a developmental time point at which remarkable increases in axonal growth and dendritic density occur and is neurodevelopmentally equivalent to a full-term human infant [22]. A global proteomic approach was used to identify differentially expressed synaptosomal proteins and key molecular network changes between the ischemic and non-ischemic hemispheres in mouse pups. Elucidating the major pathways altered by IPS will likely uncover specific mechanisms that may underlie synaptic dysfunction and uncover potential targets for preventative and therapeutic strategies.

\section{Materials and Methods}

\section{Animals}

C57B6/J mice were kept in 14-h/10-h light/dark cycle with ad libitum access to food and water.

\section{Induction of Ischemia}

The procedure was performed as previously described [23] with modifications. Pups were sexed and randomized for ischemic or sham surgery. P10 pups from litters of 7-10 pups were anesthetized with an intraperitoneal (i.p.) injection of ketamine $(100 \mathrm{mg} /$ $\mathrm{kg}$, Phoenix, St. Joseph, MO, USA) and xylazine (10 mg/kg, LLOYD Laboratories, Shenandoah, IA, USA). A small midline incision was made in the neck to isolate the right common carotid artery, which was then permanently ligated using silk. The incision was closed with a 6-0 surgical suture (Ethicon, Somerville, NJ, USA). After recovering from anesthesia on a heat pad to prevent inadvertent therapeutic hypothermia, pups were reunited with nursing dams in their home cages. Sham-operated animals underwent incision and carotid artery isolation without ligation.

\section{Body Temperature Monitoring}

Body temperature was monitored throughout the surgical procedure using a rectal temperature probe for neonatal mice (Physitemp Instruments Inc., Clifton, NJ, USA). Pup temperature was taken again $24 \mathrm{~h}$ after surgery. No significant interaction between treatment and sex or difference between groups using 2-way ANO$\mathrm{VA}$ and post hoc Bonferroni-corrected $t$ test were found (data not shown).

\section{Vibrissae-Evoked Forepaw Placement Assessment}

Five days after ischemia, pups were tested for sensorimotor function using a whisker-evoked forepaw placement as previously described [24]. In brief, pups were held by the neck skin to allow forepaw movement and at a $45^{\circ}$ angle from the bench top. They were then slowly moved upward to stimulate their whiskers by brushing against the edge of the bench top. Ten trials were performed for each side and the frequency of IL forepaw placements onto the bench surface was recorded for each animal. Two-way ANOVA was used to analyze the mean differences among groups and, where appropriate, the post hoc Bonferroni-corrected $t$ test was used to calculate mean between-group differences. Statistical analyses were performed using GraphPad Prism (GraphPad Software, Inc., La Jolla, CA, USA).

\section{Tail Suspension Test}

Five days after ischemia induction, pups were tested for motor function using a tail suspension test as previously described [24]. In brief, pups were suspended head down by their tails approximately $10-12 \mathrm{~cm}$ above the horizontal surface and then slowly lowered towards the surface. Mice normally respond by reaching toward the surface, with both forelimbs and hindlimbs extended and abducted, and dorsiflection (C-shaped bending) of the body; any deviations from these stereotypical responses were considered abnormal (Supplemental Video Recordings 1, 2; for all online suppl. material, see www.karger.com/doi/10.1159/000499126). Behavioral testers were blinded to treatment groups. The $\chi^{2}$ test for an association between ischemia and motor dysfunction was performed (GraphPad Prism). 


\section{2,3,5-Triphenyl-Tetrazolium Chloride Staining}

Brain injury was assessed with 2,3,5-triphenyl-tetrazolium chloride (TTC) staining as previously described [25]. In brief, $24 \mathrm{~h}$ or 5 days after ischemia, brains were removed from the crania and sectioned at $2.0-\mathrm{mm}$ thickness using an ice-cold mouse brain matrix (RWD Life Science, Shenzhen, China). Sections were stained with $2 \%$ TTC (diluted in PBS) at $37^{\circ} \mathrm{C}$ for $30 \mathrm{~min}$ in a 6-well plate, and subsequently fixed in $4 \%$ paraformaldehyde (in PBS) overnight at $4{ }^{\circ} \mathrm{C}$. Sections were then mounted onto a glass slide and covered with a glass coverslip and a drop of mineral oil. Images were captured using an iPhone 7 digital camera (Apple Inc.). The extent of brain injury was quantified as a ratio of infarct volume over total analyzed brain volume using Adobe Photoshop CS. Fisher's exact test for association between ischemia and brain injury was performed (GraphPad Prism).

\section{Synaptosomal Preparation}

Twenty-four hours after ischemia induction, pups were euthanized by decapitation for brain dissection. Following the removal of the cerebellum, the brain was bisected along the midline on an ice-cold metal block. Ischemic (IL) and non-ischemic (CL) hemispheres were isolated. Synaptosomes were prepared from each hemisphere following the protocol described previously [21, 26]. In brief, the ischemic and non-ischemic hemispheres were homogenized in ice-cold solution A $(0.32 \mathrm{M}$ sucrose, $1 \mathrm{mM} \mathrm{NaH}$ $\mathrm{CO}_{3}, 1 \mathrm{mM} \mathrm{MgCl}_{2}$, and $\left.0.5 \mathrm{mM} \mathrm{CaCl}_{2}\right)$ and centrifuged $(1,400 \mathrm{~g})$ at $4{ }^{\circ} \mathrm{C}$ for $10 \mathrm{~min}$. The soluble portion of the fraction was saved, while the pellet was resuspended with an additional $3 \mathrm{~mL}$ of solution A and centrifuged $(710 \mathrm{~g})$ for $10 \mathrm{~min}$. The supernatant and the previously soluble portion of the fraction were combined and centrifuged $(13,800 \mathrm{~g})$ for $10 \mathrm{~min}$. The pellet was collected and resuspended in $0.5 \mathrm{~mL}$ of ice-cold solution $\mathrm{B}(0.32 \mathrm{M}$ sucrose, 1 $\mathrm{mM} \mathrm{NaHCO}_{3}$ ) and layered onto a sucrose gradient composed of $0.85 \mathrm{M}, 1.00 \mathrm{M}$, and $1.20 \mathrm{M}$ sucrose buffered in $1 \mathrm{mM} \mathrm{NaHCO}_{3}$. This was centrifuged at $32,800 \mathrm{~g}$ for $120 \mathrm{~min}$ using a swing-bucket rotor (Beckman-Coulter, Indianapolis, IN, USA). The synaptosomal band above the $1.20 \mathrm{M}$ sucrose layer was removed from the gradient, diluted 10 -fold with solution $\mathrm{B}$, and then centrifuged at 13,800 $\mathrm{g}$ for $30 \mathrm{~min}$ to obtain the synaptosomal protein pellet. The quality of synaptosomal proteins was validated by Western blot analysis of synaptic protein enrichment (online Suppl. Fig. 1A, B).

\section{Protein Extraction}

Synaptosomes from hemispheres of 3 pups were pooled for protein preparation. Four pooled ( 2 ischemic and 2 non-ischemic) synaptosomal protein isolates were generated. Synaptosomal protein was extracted following the protocol described previously [21]. In brief, solution B was aspirated from the synaptosomal pellet and replaced with $125 \mu \mathrm{L}$ of protein extraction buffer $(7 \mathrm{M}$ urea, $2 \mathrm{M}$ thiourea, $0.4 \mathrm{M}$ triethylammonium bicarbonate [TEAB] $\mathrm{pH}$ $8.5,20 \%$ methanol, and $4 \mathrm{mM}$ tris (2-carboxyethyl) phosphine [TCEP]) on ice. The samples were then resuspended and transferred to a PCT tube with a $100-\mu \mathrm{L}$ cap for the Barocycler NEP2320 (Pressure Biosciences, South Easton, MA, USA). The samples were cycled between $35 \mathrm{kpsi}(30 \mathrm{~s})$ and $0 \mathrm{kpsi}(15 \mathrm{~s})$ for a total of 40 cycles at $37^{\circ} \mathrm{C}$. Each sample received $5 \mu \mathrm{L}$ of $200 \mathrm{~mm}$ methyl methanethiosulfonate (MMTS, $8 \mathrm{mM}$ final concentration) mixed by brief vortex, and then incubated for $15 \mathrm{~min}$ at room temperature. Each sample was then transferred to a $1.5-\mathrm{mL}$ microfuge Protein
LoBind tube (Eppendorf, Hauppauge, NY, USA). Duplicates of each sample were taken to quantify protein concentration by Bradford assay.

\section{In-Solution Digestion and iTRAQ Labeling}

Protein digestion and iTRAQ labeling were done following a previously described protocol [21]. Each sample $(100 \mu \mathrm{L})$ was brought to the same volume using $8 \mathrm{mM}$ MMTS for the iTRAQ 4-plexes analysis. Ultrapure water was used to dilute each sample (4-fold). A 1:35 ratio of trypsin (Promega, Madison, WI, USA) was added and followed by a 16 -h incubation at $37^{\circ} \mathrm{C}$, a 30 -min freeze at $-80^{\circ} \mathrm{C}$, and a dehydration in vacuo for the trypsin digestion process. Each sample was cleaned with a 4 -mL Extract Clean C18 SPE cartridge (Grace-Davidson, Deerfield, IL, USA). Eluents were vacuum-dried and resuspended in dissolution buffer of the iTRAQ kit (final concentration $2 \mu \mathrm{g} / \mu \mathrm{L}$ ). Each $40 \mu \mathrm{g}$ sample was labeled following the manufacturer's protocol. After iTRAQ labeling, samples were cleaned using a 4-mL Extract Clean C18 SPE cartridge and dried in vacuo.

\section{Offline Fractionation and Mass Spectrometry}

Fractionation and mass spectrometry were performed as described previously [21]. In brief, the iTRAQ 4-plexes sample was resuspended in buffer A ( $10 \mathrm{mM}$ ammonium formate $\mathrm{pH} 10$ in $98 \%$ water and $2 \%$ acetonitrile) and fractionated offline by high $\mathrm{pH}$ reverse-phase chromatography using MAGIC 2002 HPLC (Michrom BioResources, Auburn, CA, USA) and C18 Gemini-NX column $(150 \times 2 \mathrm{~mm}$ ID, $5-\mu \mathrm{m}$ particle, $110 \AA$ pore size [Phenomenex, Torrence, CA, USA]). Buffer B (10 mM ammonium formate $\mathrm{pH} 10$ in 10\% water and $90 \%$ acetonitrile) was used to maintain the flow rate $(100 \mu \mathrm{L} / \mathrm{min})$ at a $5-35 \%$ gradient for $60 \mathrm{~min}$ and a $35-60 \%$ gradient for $5 \mathrm{~min}$. Fractions were collected every $2 \mathrm{~min}$ and UV-light absorbance was read at 215 and $280 \mathrm{~nm}$. Fractions containing peptide were divided into 2 equal groups labeled "early" and "late." The first "early" fraction was concatenated with the first "late" fraction, and so on. Samples were then vacuum-dried and resuspended in loading solvent (98\% water, $2 \%$ acetonitrile, and $0.01 \%$ formic acid). Aliquots $(1-1.5 \mu \mathrm{L})$ were run on a Velos Orbitrap mass spectrometer (Thermo Fisher Scientific, Waltham, MA, USA). The RAW data (Proteowizard files) were converted to the MzXML format using MSconvert software and to MGF files using the TINT raw-MGF converter. Collected data were analyzed using ProteinPilot ${ }^{\mathrm{TM}} 4.5$ (AB Sciex, Foster City, CA, USA). Search parameters were: cysteine MMTS; iTRAQ 4-plexes (peptide-labeled); trypsin; instrument Orbi MS (1-3 ppm) Orbi MS/MS; bias corrections were applied to account for biological modifications including systematic errors in protein amount among samples; a thorough search effort; and local 5\% false discovery rate analysis (with reversed database). A summary of the protein search and identification is shown in online Supplementary Figure 1C.

\section{iTRAQ Data Treatment and Selection}

The 4-plexes parameter was set up as follows: $m / z 113$ (IL1), $m / z 114$ (CL1), $m / z 115$ (IL2), and $m / z 116$ (CL2). The $m / z 114$ was used as denominator (control) to generate ratio values (IL/CL). Proteins with $<3$ distinct peptides ( $95 \%$ confidence) or error factor (EF) $>2.0$ were eliminated from the analysis. These selection criteria resulted in 1,498 identified proteins from the 4-plexes dataset. Differentially expressed proteins were identified using criteria (absolute [fold change] $\geq 1.5 \times ; p<0.05$ ). 


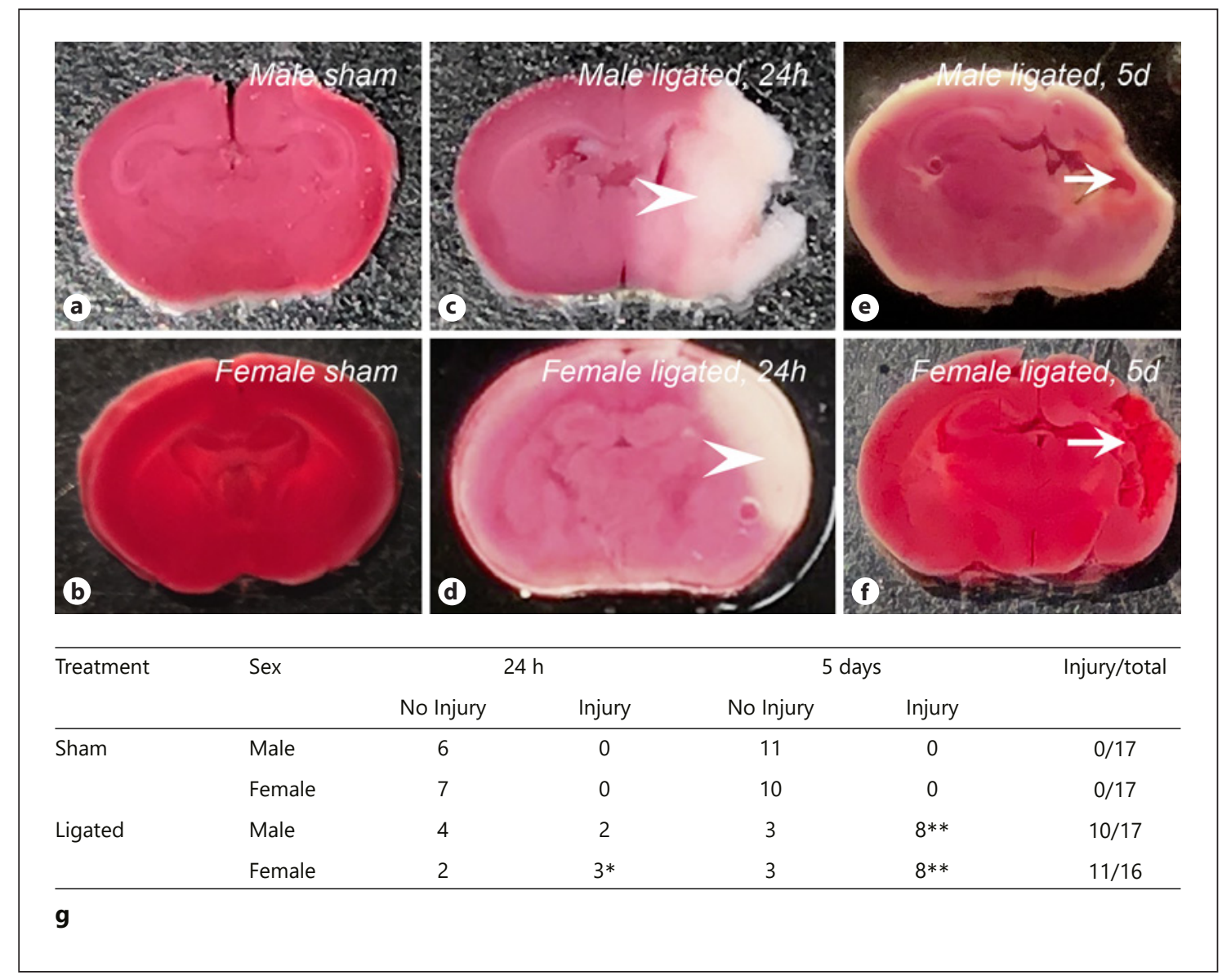

Fig. 1. P10 ischemia induced variable brain injury in mouse pups. Representative images of TTC stained brain sections from sham (a, b) and ischemic mouse pups showing injured ipsilateral hemispheres at $24 \mathrm{~h}(24 \mathrm{~h}$; c, d, arrows, white areas) and 5 days ( $5 \mathrm{~d} ; \mathbf{e}, \mathbf{f}$, arrow) after ischemia. $\mathbf{g}$ Table showing the number of pups with or without brain injury assessed by TTC following ischemia. ${ }^{* *} p<0.01,{ }^{*} p<0.05$, Fisher's exact test for association between ischemia and brain injury.

\section{Bioinformatics}

IPA was used to identify networks, pathways, molecular and cellular functions, and behavioral and neurological dysfunctions using proteins from the iTRAQ datasets. Fisher's exact test was used to calculate the $p$ value for the proteins in the datasets that mapped onto specific pathways and functions. IPA does this by using an algorithm based on molecular function, cellular function, and functional group.

\section{Western Blot Analysis}

Western blot was used to quantify the levels of selected proteins chosen from 72 differentially expressed proteins. Protein lysates ( $n=4-7$ /group) prepared from ischemic (IL), non-ischemic (CL), and sham control hemispheres were separated using a 4-12\% BisTris gel (Novex, Life Technologies, Carlsbad, CA, USA), and transferred to a nitrocellulose membrane (Pierce, Rockford, IL, USA). The membranes were blocked using a blocking buffer for fluorescence Western blotting (Rockland, Gilbertsville, PA, USA) and sealed in a plastic bag. The membranes were treated with primary antibodies and then with fluorescent secondary antibodies. Primary antibodies included: CaMKIIa (1:2,000, Abcam, Cambridge, MA, USA), MAP-2 (1:2,000, Abcam), MAPK (1:1,000, Cell Signaling, Danvers, MA, USA), phospho-PKCa (1:1,000, Cell Signaling), PKCa (1:1,000, Cell signaling), PSD-95 (1:2,000, Abcam), $\alpha$-synuclein (1:1,000, Abcam); and $\beta$-actin (1:1,000, Sigma, St. Louis, MO, USA). Secondary antibodies included: DyLight 700 anti-mouse (1:12,500×, Rockland) and DyLight 800 anti-rabbit $(1: 12,500 \times$, Rockland). The membranes were then analyzed by near-infrared fluorescence using the Odyssey infrared imaging system (Li-Cor Biotechnology, Lincoln, NE, USA). The protein of interest was normalized against $\beta$-actin and quantified using ImageStudio software (Li-Cor). Representative Western blot images are shown in online Supplementary Figure 2. One-way ANOVA was used to analyze the mean differences among groups, and where appropriate, the post hoc Tukey $t$ test was used to calculate the mean difference between groups. Statistical analyses were performed using GraphPad Prism. 
Fig. 2. Impaired sensorimotor and motor function in mouse pups 5 days after ischemia. a Graph showing reduced frequency of forelimb extension following vibrissae stimulation contralateral to ligation in male pups compared to sham controls. Values are mean \pm SEM. $n=12-13$ males and 10 females. ANOVA, significant difference between groups with ${ }^{* * *} p<0.001$, post hoc Bonferroni-corrected $t$ test. b-d Abnormal motor function in mouse pups 5 days after ischemia. Representative still images of a mouse pup showing an attempt of dorsiflection (b, curved line) and hindlimb hemiparesis (c, arrow) ipsilateral to the ligation. d Table showing results of tail suspension test of ischemic pups performed 5 days after ligation. $\chi^{2}$ test for an association between ischemia and motor dysfunction with significant differences between sham and ligated groups with ${ }^{* *} p<0.01, * p<$ 0.05. IL, ipsilateral hemisphere; CL, contralateral hemisphere.
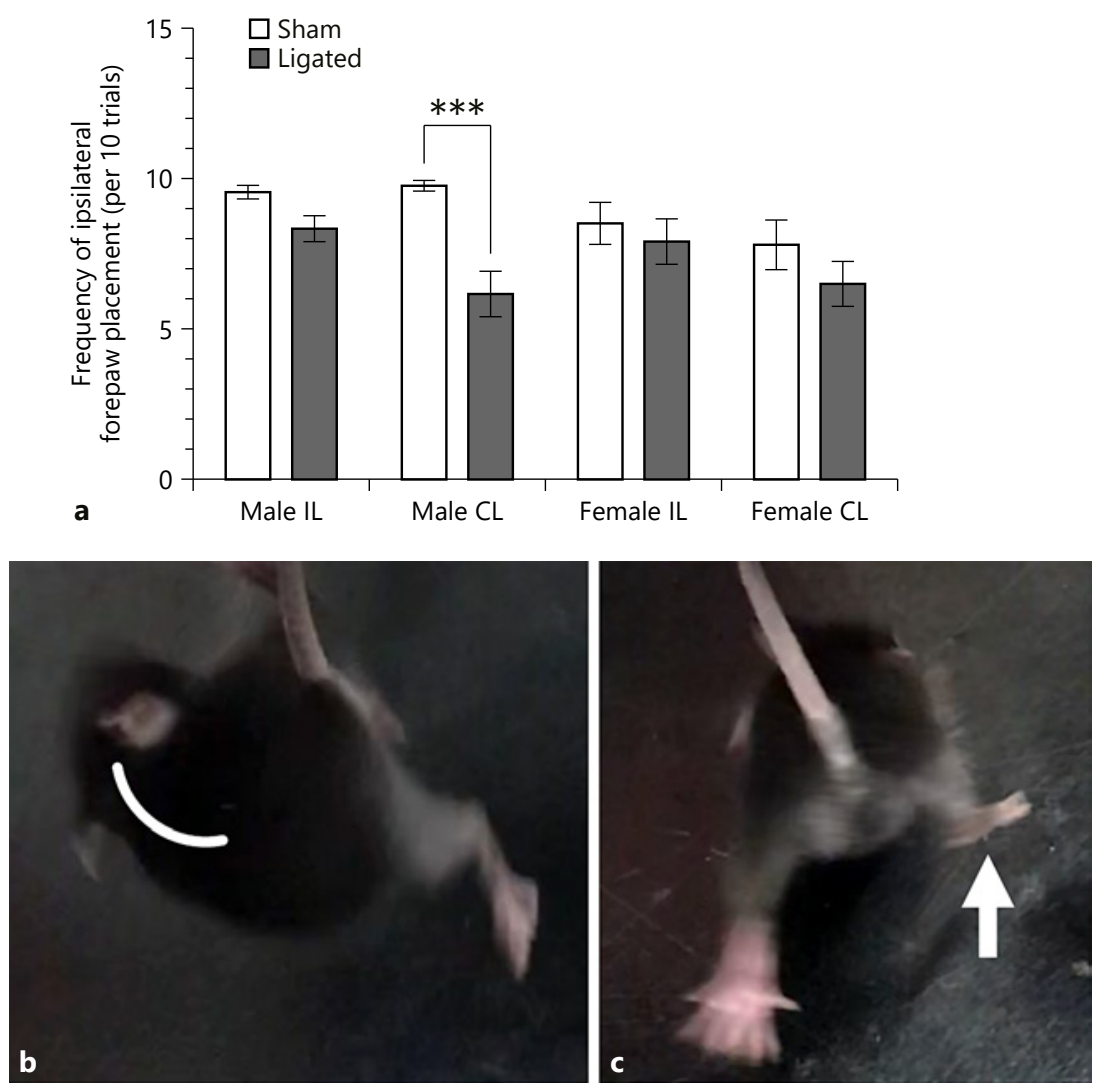

\begin{tabular}{llccccccc}
\hline Treatment & Sex & \multicolumn{3}{c}{ Dorsiflection } & \multicolumn{2}{c}{ Hindlimb hemiparesis } & Total, $n$ \\
& & $\mathrm{CL}=\mathrm{IL}$ & $\mathrm{CL}<\mathrm{IL}$ & $\mathrm{CL}>\mathrm{IL}$ & $\mathrm{CL}$ & $\mathrm{IL}$ & None & \\
\hline \multirow{2}{*}{ Sham } & Male & 10 & 1 & 2 & 1 & 0 & 12 & 13 \\
& Female & 5 & 2 & 3 & 0 & 0 & 10 & 10 \\
\multirow{3}{*}{ Ligated } & Male & 2 & $10 * *$ & 0 & 0 & $6 *$ & 6 & 12 \\
& Female & 5 & 4 & 1 & 0 & 3 & 7 & 10 \\
\hline
\end{tabular}

d

\section{Results}

\section{Perinatal Ischemia Induces IL Brain Injury}

Accompanied by Sensorimotor and Motor

Impairments

TTC staining was used to visualize brain injury following ischemia induced by ligation of the right common carotid artery in P10 mouse pups. Assessments at $24 \mathrm{~h}$ and 5 days after ischemia showed brain injury IL to the ligation in both male and female pups when compared with sham-operated pups. The extent of injury ranged from no visible injury to about $27 \%$ of total brain volume, with injury still appreciable 5 days after ischemia (Fig. 1).
To further demonstrate the functional consequence of perinatal ischemia, 5 days after ischemia, the sensorimotor and motor functions were assessed by vibrissae-evoked forelimb placement and the tail suspension analysis, respectively. Ischemic male pups showed a lower frequency of forepaw placement CL to the ligation side when compared with sham controls (Fig. 2a; $p=0.032$ sex difference). In addition, ischemic male pups showed greater attempts of dorsiflection of the body, favoring the ligated hemisphere (Fig. 2b, d). A significant number of ischemic male pups showed hindlimb hemiparesis IL to the ligation when compared to the sham control group (Fig. 2c, d). 
Fig. 3. IPA-mapped differentially expressed synaptosomal proteins in P10 mouse pups $24 \mathrm{~h}$ after ischemia onto molecular networks associated with decreased (a) and increased (b) functions or diseases. Red shapes, upregulated; green shapes, downregulated; blue shapes, predicted inhibition; orange shapes, predicted activation; blue arrows, activation; orange arrows, inhibition; yellow arrows, findings inconsistent with state of downstream molecule; gray arrows, effects not predicted.

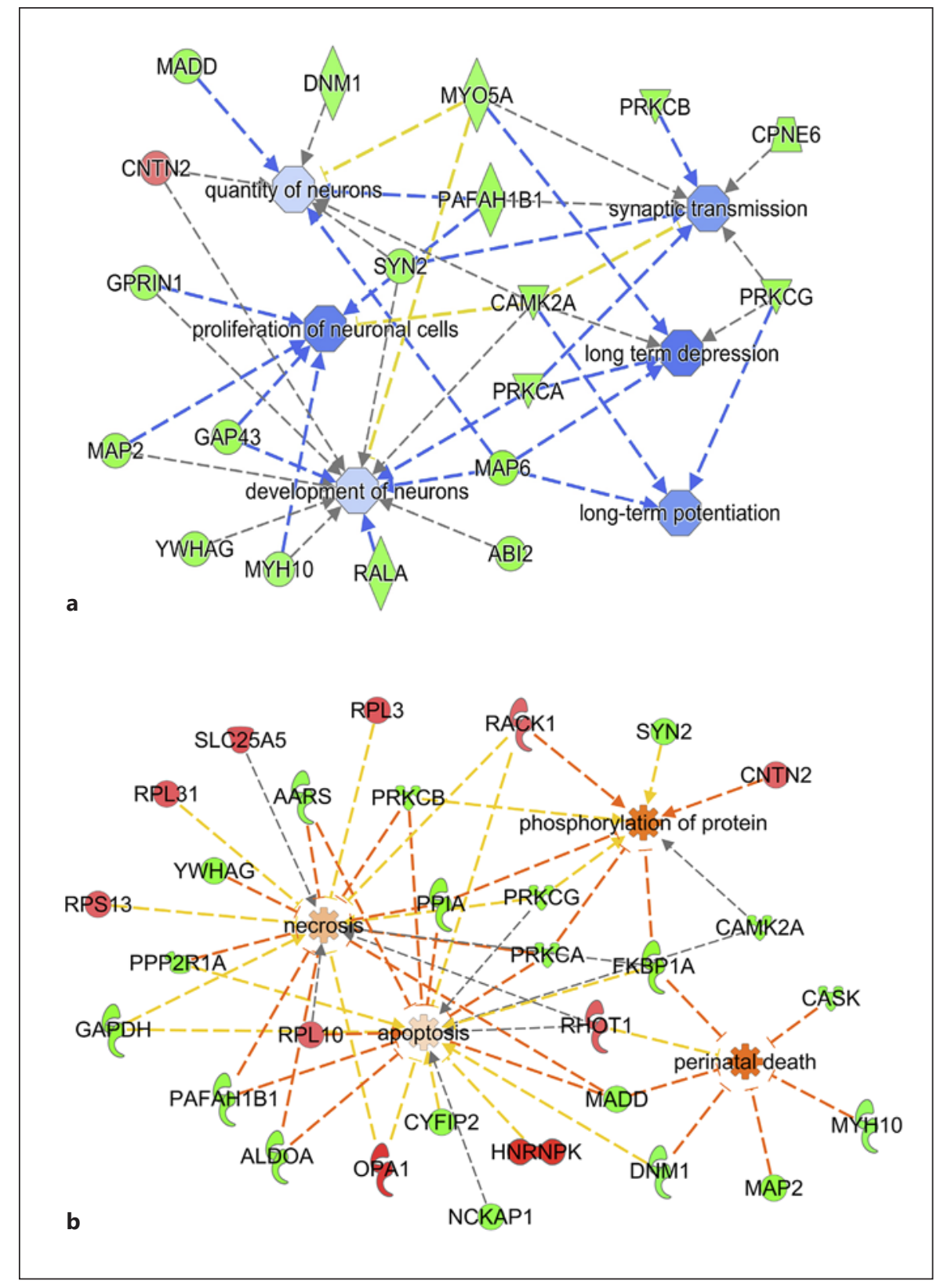

Perinatal Ischemia Suppresses Interactive Gene Networks Implicated in Neuronal Development and Survival

Proteomic analysis showed that 72 of 1,498 qualified proteins $(4.8 \%)$ were differentially expressed (absolute [fold change] $\geq 1.5 \times ; p<0.05$ ) between the ischemic and non-ischemic hemispheres (Table 1), including developmentally relevant synaptic proteins (GAP43, synapsin II, and CASK) and energetically important mitochondrial proteins (Cox7c, SLC25A5, and NDUFA4). IPA mapped these differentially expressed proteins onto networks that predict reduced neuronal proliferation and synaptic plasticity concomitant with increased phosphorylation of protein, perinatal death, and neuronal cell death (Fig. 3). Using IPA, differentially expressed synaptosomal proteins were also used to predict altered activities of upstream regulators (Table 2). Inhibition of inflammatory regulators (NFE2L2 and ADORA2A) and activation of neuronal growth and differentiation regulators (ESRRa and mTOR) were the significant perinatal ischemic ef- 
Table 1. Differentially-expressed synaptosomal proteins in ischemic hemisphere $24 \mathrm{~h}$ after unilateral common carotid artery ligation of P10 mice

\begin{tabular}{|c|c|c|c|c|c|}
\hline \multirow[t]{2}{*}{ Gene name } & \multirow[t]{2}{*}{ Symbol } & \multicolumn{2}{|c|}{$\begin{array}{l}\text { Ischemic/ } \\
\text { non-ischemic }\end{array}$} & \multirow[t]{2}{*}{ Location } & \multirow[t]{2}{*}{ Type(s) } \\
\hline & & $\log _{2}(\mathrm{FC})$ & $p$ value & & \\
\hline abl-interactor 2 & $\mathrm{ABI} 2$ & -1.265 & 0.047 & cytoplasm & other \\
\hline acyl-CoA oxidase 1 , palmitoyl & ACOX1 & 1.261 & 0.039 & cytoplasm & enzyme \\
\hline alanyl-tRNA synthetase & AARS & -1.149 & 0.004 & cytoplasm & enzyme \\
\hline aldehyde dehydrogenase 1 family, member L1 & ALDH1L1 & -1.130 & 0.027 & cytoplasm & enzyme \\
\hline aldo-keto reductase family 1 , member Al (aldehyde reductase) & AKR1Al & -1.310 & 0.015 & cytoplasm & enzyme \\
\hline aldolase A, fructose-bisphosphate & ALDOA & -1.256 & 0.000 & cytoplasm & enzyme \\
\hline asparaginase-like 1 & ASRGL1 & -1.288 & 0.049 & cytoplasm & enzyme \\
\hline ATP synthase, $\mathrm{H}+$ transporting, mitochondrial Fo complex, subunit B1 & ATP5F1 & 1.261 & 0.032 & cytoplasm & transporter \\
\hline ATP synthase, $\mathrm{H}+$ transporting, mitochondrial Fo complex, subunit $\mathrm{G}$ & ATP5L & 1.376 & 0.022 & cytoplasm & transporter \\
\hline ATPase, $\mathrm{H}+$ transporting, lysosomal $31 \mathrm{kDa}, \mathrm{V} 1$ subunit $\mathrm{E} 1$ & ATP6V1E1 & -1.157 & 0.036 & cytoplasm & transporter \\
\hline ATPase, $\mathrm{H}+$ transporting, lysosomal $34 \mathrm{kDa}, \mathrm{V} 1$ subunit $\mathrm{D}$ & ATP6V1D & -1.155 & 0.044 & cytoplasm & transporter \\
\hline ATPase, $\mathrm{H}+$ transporting, lysosomal $42 \mathrm{kDa}, \mathrm{V} 1$ subunit $\mathrm{C} 1$ & ATP6V1C1 & -1.131 & 0.034 & cytoplasm & transporter \\
\hline ATPase, $\mathrm{H}+$ transporting, lysosomal $56 / 58 \mathrm{kDa}$, V1 subunit $\mathrm{B} 2$ & ATP6V1B2 & -1.079 & 0.050 & cytoplasm & transporter \\
\hline ATPase, $\mathrm{H}+$ transporting, lysosomal 70kDa, $\mathrm{V} 1$ subunit A & ATP6V1A & -1.076 & 0.037 & plasma membrane & transporter \\
\hline calcium/calmodulin-dependent protein kinase II alpha & CAMK2A & -1.227 & 0.003 & cytoplasm & kinase \\
\hline calcium/calmodulin-dependent serine protein kinase (MAGUK family) & CASK & -1.128 & 0.043 & plasma membrane & kinase \\
\hline CaM kinase-like vesicle-associated & CAMKV & -1.224 & 0.004 & other & kinase \\
\hline contactin 2 (axonal) & CNTN2 & 1.240 & 0.000 & plasma membrane & other \\
\hline copine VI (neuronal) & CPNE6 & -1.230 & 0.016 & plasma membrane & transporter \\
\hline cytochrome $\mathrm{c}$ oxidase subunit VIIc & $\operatorname{Cox} 7 \mathrm{c}$ & 1.443 & 0.048 & cytoplasm & other \\
\hline cytoplasmic FMR1 interacting protein 2 & CYFIP2 & -1.198 & 0.014 & cytoplasm & other \\
\hline dimethylarginine dimethylaminohydrolase 1 & DDAH1 & -1.243 & 0.017 & cytoplasm & enzyme \\
\hline dynamin 1 & DNM1 & -1.080 & 0.034 & cytoplasm & enzyme \\
\hline erythrocyte membrane protein band 4.1-like 1 & EPB41L1 & -1.279 & 0.001 & plasma membrane & other \\
\hline erythrocyte membrane protein band 4.1-like 2 & EPB41L2 & -1.216 & 0.007 & plasma membrane & other \\
\hline FK506 binding protein $1 \mathrm{~A}, 12 \mathrm{kDa}$ & FKBP1A & -1.489 & 0.042 & cytoplasm & enzyme \\
\hline G protein regulated inducer of neurite outgrowth 1 & GPRIN1 & -1.163 & 0.007 & plasma membrane & other \\
\hline G-protein signaling modulator 1 & GPSM1 & -1.447 & 0.046 & cytoplasm & other \\
\hline glutamate-ammonia ligase & GLUL & -1.162 & 0.003 & cytoplasm & enzyme \\
\hline glyceraldehyde-3-phosphate dehydrogenase & GAPDH & -1.320 & 0.000 & cytoplasm & enzyme \\
\hline growth associated protein 43 & GAP43 & -1.277 & 0.026 & plasma membrane & other \\
\hline guanine nucleotide binding protein ( $\mathrm{G}$ protein), beta polypeptide 2 -like 1 & GNB2L1 & 1.216 & 0.040 & cytoplasm & enzyme \\
\hline heterogeneous nuclear ribonucleoprotein $\mathrm{K}$ & HNRNPK & 1.700 & 0.006 & nucleus & other \\
\hline lipid phosphate phosphatase-related protein type 3 & LPPR3 & -1.189 & 0.040 & other & phosphatase \\
\hline MAP-kinase activating death domain & MADD & -1.179 & 0.045 & cytoplasm & other \\
\hline MARCKS-like 1 & MARCKSL1 & -1.179 & 0.035 & cytoplasm & other \\
\hline microtubule-associated protein 2 & MAP2 & -1.257 & 0.001 & plasma membrane & other \\
\hline microtubule-associated protein 6 & MAP6 & -1.433 & 0.001 & cytoplasm & other \\
\hline myosin VA (heavy chain 12 , myoxin) & MYO5A & -1.070 & 0.036 & cytoplasm & enzyme \\
\hline myosin, heavy chain 10 , non-muscle & MYH10 & -1.069 & 0.028 & cytoplasm & other \\
\hline NCK-associated protein 1 & NCKAP1 & -1.178 & 0.003 & plasma membrane & other \\
\hline NDUFA4, mitochondrial complex associated & NDUFA4 & 1.548 & 0.034 & cytoplasm & enzyme \\
\hline optic atrophy 1 (autosomal dominant) & OPAl & 1.608 & 0.030 & cytoplasm & enzyme \\
\hline OTU domain, ubiquitin aldehyde binding 1 & Otubl & -1.217 & 0.045 & cytoplasm & enzyme \\
\hline peptidylprolyl isomerase A (cyclophilin A) & PPIA & -1.394 & 0.003 & cytoplasm & enzyme \\
\hline phosphofructokinase, liver & PFKL & -1.151 & 0.048 & cytoplasm & kinase \\
\hline phosphorylase, glycogen; brain & PYGB & -1.117 & 0.001 & cytoplasm & enzyme \\
\hline platelet-activating factor acetylhydrolase $1 \mathrm{~b}$, regulatory subunit 1 (45 kDa) & PAFAH1B1 & -1.226 & 0.000 & cytoplasm & enzyme \\
\hline protein kinase $\mathrm{C}$, alpha & PRKCA & -1.181 & 0.015 & cytoplasm & kinase \\
\hline protein kinase $C$, beta & PRKCB & -1.262 & 0.010 & cytoplasm & kinase \\
\hline protein kinase $\mathrm{C}$, gamma & PRKCG & -1.283 & 0.002 & cytoplasm & kinase \\
\hline protein phosphatase 2 , regulatory subunit $\mathrm{A}$, alpha & PPP2R1A & -1.175 & 0.021 & cytoplasm & phosphatase \\
\hline RAB11B, member RAS oncogene family & RAB11B & -1.147 & 0.025 & cytoplasm & enzyme \\
\hline RAB6A, member RAS oncogene family & RAB6A & -1.220 & 0.025 & cytoplasm & enzyme \\
\hline ras homolog family member $\mathrm{T} 1$ & RHOT1 & 1.277 & 0.042 & cytoplasm & enzyme \\
\hline RAS protein activator like 1 (GAP1-like) & RASAL1 & -1.250 & 0.015 & cytoplasm & other \\
\hline ribosomal protein $\mathrm{L} 10$ & RPL10 & 1.224 & 0.030 & cytoplasm & other \\
\hline ribosomal protein $\mathrm{L} 12$ & RPL12 & 1.284 & 0.021 & nucleus & other \\
\hline ribosomal protein L3 & RPL3 & 1.322 & 0.015 & cytoplasm & other \\
\hline ribosomal protein $\mathrm{L} 31$ & RPL31 & 1.295 & 0.023 & cytoplasm & other \\
\hline ribosomal protein $\mathrm{S} 13$ & RPS13 & 1.254 & 0.026 & other & other \\
\hline ribosomal protein S25 & RPS25 & 1.477 & 0.046 & cytoplasm & other \\
\hline septin 7 & SEPT7 & -1.133 & 0.039 & cytoplasm & other \\
\hline signal recognition particle receptor, B subunit & SRPRB & 1.259 & 0.001 & cytoplasm & other \\
\hline $\begin{array}{l}\text { solute carrier family } 25 \text { (mitochondrial carrier; adenine nucleotide translocator), } \\
\text { member } 5\end{array}$ & SLC25A5 & 1.349 & 0.004 & cytoplasm & transporter \\
\hline synapsin II & SYN2 & -1.175 & 0.013 & plasma membrane & other \\
\hline talin 2 & TLN2 & -1.161 & 0.041 & nucleus & other \\
\hline translin & TSN & -1.312 & 0.032 & nucleus & other \\
\hline tubulin, alpha $4 \mathrm{a}$ & TUBA4A & -1.242 & 0.017 & cytoplasm & other \\
\hline tyrosine 3-monooxygenase/tryptophan 5-monooxygenase activation protein, gamma & YWHAG & -1.227 & 0.005 & cytoplasm & other \\
\hline v-ral simian leukemia viral oncogene homolog A (ras-related) & RALA & -1.165 & 0.025 & cytoplasm & enzyme \\
\hline vesicle amine transport 1-like & VAT1L & -1.303 & 0.019 & other & enzyme \\
\hline
\end{tabular}


Table 2. Top 5 upstream regulators induced by ischemia in P10 mouse CNS

\begin{tabular}{llllll}
\hline $\begin{array}{l}\text { Upstream } \\
\text { regulator }\end{array}$ & Molecule type & $\begin{array}{l}\text { Predicted } \\
\text { activation state }\end{array}$ & $\begin{array}{l}\text { Activation } \\
z \text { score }\end{array}$ & $\begin{array}{l}p \text { value } \\
\text { of overlap }\end{array}$ & Target molecules in dataset \\
\hline ESRRa & transcription regulator & activated & 2.24 & $4.79 \times 10^{-6}$ & ACOX1, ALDOA, ATP5F1, Cox7c, PFKL \\
\hline ADORA2A & G-protein coupled receptor & inhibited & -1.63 & $1.80 \times 10^{-6}$ & $\begin{array}{l}\text { ATP6V1B2, CAMKV, GAPDH, HNRNPK, } \\
\text { PPP2R1A, RAB6A }\end{array}$ \\
\hline mTOR & kinase & & -1.13 & $4.74 \times 10^{-7}$ & $\begin{array}{l}\text { AKR1A1, ATP5F1, GAP43, Otub1, PYGB, } \\
\text { RAB11B, RPL12 }\end{array}$ \\
\hline NFE2L2 & transcription regulator & inhibited & -1.89 & $7.71 \times 10^{-5}$ & $\begin{array}{l}\text { AKR1A1, ALDOA, GNB2L1, NCKAP1, } \\
\text { PAFAH1B1, PRKCB, SYN2 }\end{array}$ \\
\hline HDAC4 & transcription regulator & & & $5.77 \times 10^{-6}$ & CAMK2A, CYFIP2, PRKCA, PRKCB, SYN2 \\
\hline
\end{tabular}

Table 3. Top 5 causal network regulators altered by ischemia in P10 mouse CNS

\begin{tabular}{|c|c|c|c|c|c|c|c|}
\hline $\begin{array}{l}\text { Master } \\
\text { regulator }\end{array}$ & $\begin{array}{l}\text { Molecule } \\
\text { type }\end{array}$ & $\begin{array}{l}\text { Participating } \\
\text { regulators }\end{array}$ & $\begin{array}{l}\text { Predicted } \\
\text { activation } \\
\text { state }\end{array}$ & $\begin{array}{l}\text { Activation } \\
z \text { score }\end{array}$ & $\begin{array}{l}p \text { value } \\
\text { of overlap }\end{array}$ & $\begin{array}{l}\text { Network } \\
\text { bias-cor- } \\
\text { rected } p \\
\text { value }\end{array}$ & Target molecules in dataset \\
\hline CD38 & enzyme & $\begin{array}{l}\text { CBL, CD38, CD3E, GF1R, ITK, } \\
\text { MAP2K1, Mapk, MTOR, PPARG, } \\
\text { RXRA, SERPINE1, SIRT1, TGFB1, } \\
\text { TP53 }\end{array}$ & inhibited & -2.20 & $9.88 \times 10^{-9}$ & 0.001 & $\begin{array}{l}\text { ACOX1, AKR1A1, ALDH1L1, AL- } \\
\text { DOA, ATP5F1, ATP5L, ATP6V1D, } \\
\text { CYFIP2, FKBP1A, GAP43, GAPDH, } \\
\text { GLUL, MYH10, NDUFA4, Otub1, } \\
\text { PFKL, PRKCA, PRKCB, PRKCG, } \\
\text { PYGB, RAB11B, RACK1, RPL12, } \\
\text { RPS25, VAT1L }\end{array}$ \\
\hline MAP2K1 & kinase & $\begin{array}{l}\text { CDKN1B, Creb, CTNNB1, ELK1, } \\
\text { ERK, HIF1A, INSR, MAP2K1, } \\
\text { MAPK3, MAPK7, MAPK8, } \\
\text { MAPK9, MTOR, NFE2L2, } \\
\text { NR3C1, PPARG, RPS6KA3, } \\
\text { SMAD3, TP53 }\end{array}$ & inhibited & -2.27 & $9.89 \times 10^{-9}$ & 0.001 & $\begin{array}{l}\text { ABI2, AKR1A1, ALDH1L1, ALDOA, } \\
\text { ATP5F1, ATP5L, ATP6V1D, CYFIP2, } \\
\text { FKBP1A, GAP43, GAPDH, GLUL, } \\
\text { MARCKSL1, MYH10, NCKAP1, } \\
\text { NDUFA4, Otub1, PAFAH1B1, PRK- } \\
\text { CA, PRKCB, PRKCG, PYGB, RAB11B, } \\
\text { RACK1, RPL12, RPS25, SYN2, } \\
\text { TUBA4A }\end{array}$ \\
\hline DRD3 & $\begin{array}{l}\text { G-protein } \\
\text { coupled } \\
\text { receptor }\end{array}$ & $\begin{array}{l}\text { AKT1, DRD3, ELK1, FOXO1, } \\
\text { HMOX1, MAPK1, MAPK3, } \\
\text { MTOR, NFE2L2, TP53, TSC2 }\end{array}$ & inhibited & -2.29 & $4.56 \times 10^{-8}$ & 0.001 & $\begin{array}{l}\text { AKR1A1, ALDOA, ATP5F1, AT- } \\
\text { P6V1D, CYFIP2, FKBP1A, GAPDH, } \\
\text { GLUL, MYH10, NCKAP1, NDUFA4, } \\
\text { Otub1, PAFAH1B1, PRKCA, PRKCB, } \\
\text { PRKCG, PYGB, RAB11B, RACK1, } \\
\text { RPL12, RPS25, SLC25A5, SYN2 }\end{array}$ \\
\hline Gsk3 & group & $\begin{array}{l}\text { Akt, ELK1, ERK1/2, FLI1, FOXO1, } \\
\text { Gsk3, IGF1R, MAP2K1, MAP2K2, } \\
\text { MAPT, MMP2, MTOR, NFE2L2, } \\
\text { NMDA Receptor, PRKAA1, } \\
\text { PRKCD, SLC29A1, STAT1, TP53, } \\
\text { TSC2 }\end{array}$ & activated & 2.27 & $1.29 \times 10^{-8}$ & 0.001 & $\begin{array}{l}\text { AKR1A1, ALDH1L1, ATP5F1, ATP5L, } \\
\text { ATP6V1D, CAMK2A, CYFIP2, } \\
\text { DDAH1, FKBP1A, GAP43, GAPDH, } \\
\text { GLUL, MAP2, MYH10, NDUFA4, } \\
\text { Otub1, PAFAH1B1, PPP2R1A, PRK- } \\
\text { CA, PRKCB, PRKCG, PYGB, RAB11B, } \\
\text { RACK1, RPL12, RPL3, RPS25, SYN2 }\end{array}$ \\
\hline
\end{tabular}


fects observed. Analysis of causal network regulators also indicated increased activity of VEGFa and Gsk3 accompanied by decreased activity of CD38, MAP2K1 and DRD3 (Table 3).

Perinatal Ischemia Reduces the Expression of Synaptic Proteins in Signaling Pathways Critical for Synaptic

Development

IPA mapped differentially expressed proteins onto specific canonical signaling pathways, indicating decreased activity in 4 important clusters: chemokine signaling, cAMP response element-binding protein (CREB) signaling, neuregulin signaling, and $\alpha$-adrenergic signaling (Fig. 4a). Given the documented role of CREB signaling in neonatal models of hypoxic-ischemic (HI) brain injury $[27,28]$, the differentially expressed synaptosomal proteins within the CREB signaling pathway were chosen for validation using Western blot (Fig. 4b, c). These particular proteins were also chosen because of their important role in multiple signaling pathways (e.g., CREB and mTOR). Total PKCa and its phosphorylated levels were lower in the ischemic hemisphere in male, but not female, pups (Fig. 4c). The sham-operated group of mice was used as a control and showed that ischemia induced a significant reduction of the analyzed synaptic proteins even in the non-ischemic (CL) hemisphere of mouse pups (Fig. 4c).

\section{Discussion}

Approximately $60 \%$ of IPS survivors develop longterm neurological and cognitive disabilities [1-3]. Molecular mechanisms, at the global proteomic level, that underlie IPS-associated neurodevelopmental disorders remain understudied. Our proteomic analysis of synaptosomal proteins from an IPS mouse model identified molecular pathways/networks that are critical for neuronal survival and function. The results corroborate previously documented effects of ischemic stroke on dendritogenesis $[29,30]$, neuronal death, and neuronal proliferation [31-36]. In addition, they implicate, for the first time, cellular dysfunctions including neuronal differentiation, synaptogenesis, and protein phosphorylation (Fig. 3) as potential contributing factors to the neurodevelopmental deficits following perinatal ischemic brain injury.

The finding of increased phosphorylation of protein following neonatal ischemia highlights a common molecular mechanism underlying the pathophysiology of brain injury, as phosphorylation of protein kinases was demonstrated in both adult and perinatal models of HI brain injury [37-41]. The cellular effects of increased protein phosphorylation remain to be established in this IPS model but are likely to regulate neuronal death and survival as observed in previous studies on $\mathrm{HI}$ brain injury [40-43]. Moreover, our finding of reduced $\alpha$-adrenergic and CREB signaling, activities which are regulated by protein phosphorylation and play critical roles in neuronal survival by regulating brain-derived neurotrophic factor [33, 44-46], further underscores the appropriate regulation of neuronal survival and death as a part of the repair mechanism following perinatal brain injury [27, 47-50]. Our perinatal ischemic brain injury model also showed lowered expression and signaling of $\mathrm{PKCa}$, CaMKIIa, and MAP-2, which play critical roles in longterm potentiation and depression, indicating impaired synaptogenesis and neuronal connectivity in the developing ischemic brain $[51,52]$. Reduced synaptogenesis and synaptic plasticity as predicted by IPA following perinatal ischemia are consistent with previous findings of decreased myelination $[29,30,53,54]$ which affects processing speed and neuronal differentiation [34-36]. These effects likely result in compromised neuronal connectivity. Alternatively, they may simply reflect a developmental pause in axonal and synaptic growth to minimize aberrant connectivity immediately following ischemia. Additional analyses to determine whether these signaling changes remain permanent or recover later after ischemia will be highly relevant.

Nonetheless, our findings uncover potentially novel molecular networks to explain the impaired neurocognitive development in perinatal ischemia survivors. Our findings are in line with a recent analysis of altered synaptosomal proteins due to neonatal hypoxia-ischemia, which is likely a more severe neonatal brain injury model than our ischemia model [18]. In particular, the significant decrease in synaptic proteins at $24 \mathrm{~h}$ following a transient increase of these proteins immediately following HI, that was observed in Shao et al. [18], suggests that our models of ischemia only and hypoxia-ischemia share common pathways responsible for neuronal injury and repair. Our findings of ischemic effects on the CL (nonischemic) hemisphere are also consistent with models of HI brain injury [55].

The discovery of predicted dysfunction of upstream regulators (e.g., ESRR $\alpha$ and mTOR) suggests that these factors are important targets for therapeutic manipulation to mitigate the long-term neurodevelopmental effects of IPS. While data are limited, emerging evidence 


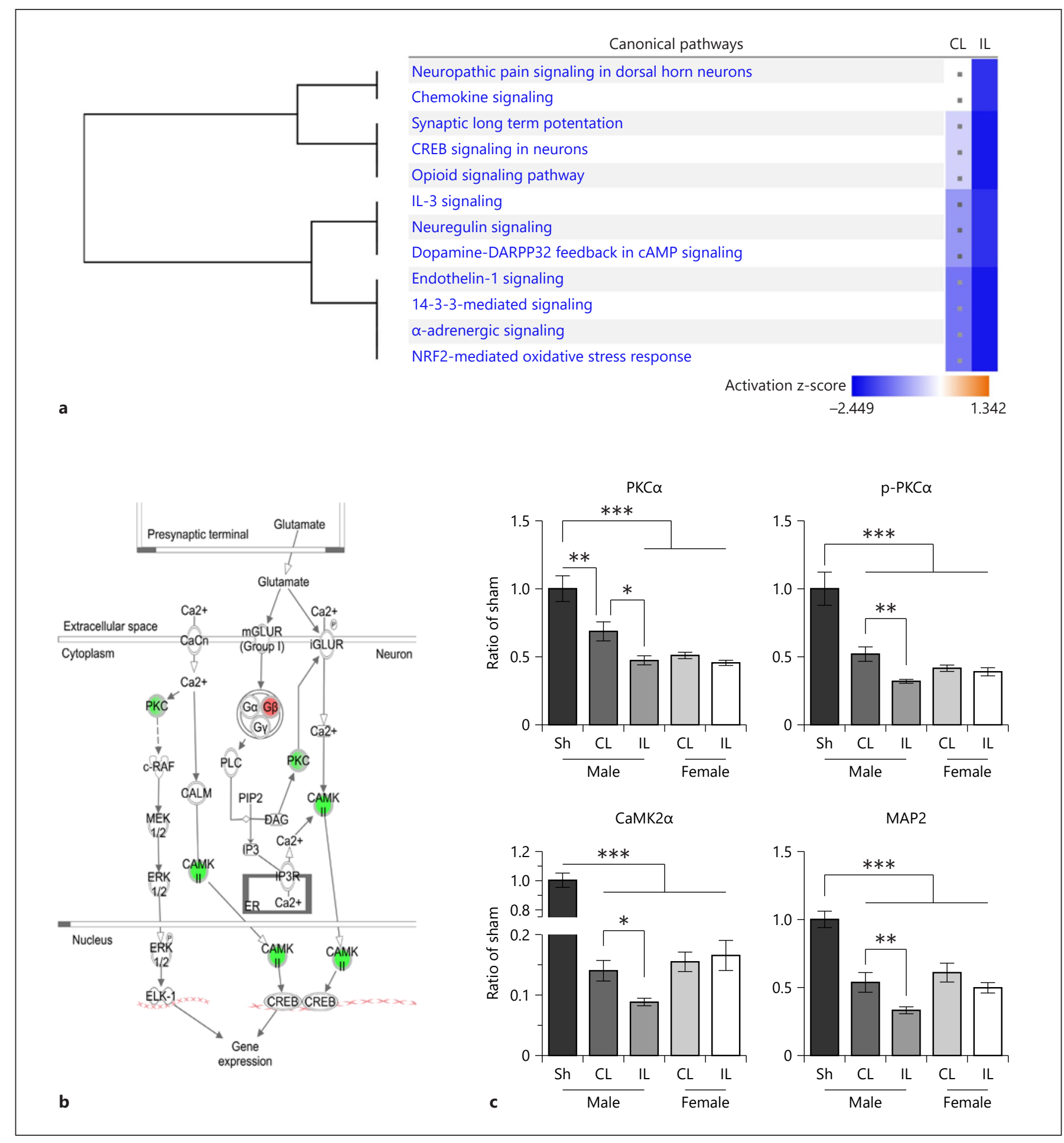

Fig. 4. IPA-annotated canonical signaling pathways altered in P10 ischemic mouse brains. a Reduced activity (blue) of 4 unique clusters of signaling pathways with a $z$ score $\leq-2.0$ in the ipsilateral (IL) versus contralateral (CL) hemispheres. Dots indicate a nonsignificant $(z$ score $>-2.0)$ activation state. $\mathbf{b}$ Altered expression of molecules within the CREB signaling pathway (green, downregulated; red, upregulated). c Validation of synaptic proteins by Western

Neonatal Ischemia Alters Synaptosomal Proteome blotting. Graphs show Western blot quantification of selected proteins in both male and female pups normalized to the male sham control (Sh) group. Values are mean \pm SEM, $n=4-7$ /group, significant difference between groups with $* p<0.05,{ }^{* *} p<0.01$, *** $p<0.001$, ANOVA with the post hoc Tukey $t$ test. p-PKCa, phosphorylated PKCa. 
links estrogen receptor and mTOR signaling to the risk of ischemic stroke and recovery [56-59]. Interestingly, given the sex-dependent incidence and outcomes of neonatal ischemic stroke in humans [7-9], our findings implicate estrogen receptor signaling and estrogen-related receptor signaling as major mechanisms that confer neuroprotection in females, lessening their vulnerability to adverse outcomes following IPS [60,61]. While measuring estrogen-related activity was beyond the scope of our study, the prediction of increased ESRRa signaling can inform future investigations of the crosstalk between ESRRa and the $\mathrm{PKC} / \mathrm{mTOR} / \mathrm{AKT} / \mathrm{MAP}-2$ signaling pathways in the etiology of neonatal ischemic stroke [62, 63]. Likewise, the targeting of inflammatory and epigenetic regulators is emerging as a novel neuroprotective strategy against brain injury in early postnatal life [6469]. In particular, therapeutic strategies targeting CD38, GSK3, and NFE2L2 signaling will likely deliver favorable outcomes, given the activation of microglia in models of neonatal brain injury $[31,53,70-72]$. Finally, the decreased dopamine $\mathrm{D} 3$ receptor (DRD3) signaling in our perinatal ischemic brain injury model is a new finding (Table 3). Activation of DRD3 signaling is known to facilitate protective effects from ischemic-reperfusion injury in the renal tissue [73]. An additional study of DRD3 signaling may lead to new insights into the role of DRD3 signaling in IPS pathophysiology.

Our study has 2 limitations. First, the inconsistent outcomes of brain injury induced by ischemia likely resulted in an underestimation of differentially expressed synaptosomal proteins, thereby limiting the scope of the findings. We were not able to address this problem as it was necessary to pool synaptosomes from 3 mouse pups to generate sufficient proteins for iTRAQ analysis. This limitation can be overcome in future studies when advancements in mass spectrometry technology allow a higher level of protein resolution and a lower protein quantity requirement, thus enabling iTRAQ analysis at the level of individual animal. Second, the analysis targeted the alteration of synaptosomal proteins within $24 \mathrm{~h}$ after ischemia, which aims to identify changes that occur during the critical period for instituting therapeutic interventions. It remains unknown whether these changes persist into adulthood. Such an analysis is also beyond the scope of this study but can be addressed in the future.

In conclusion, despite the inherent limitations, the proteomic analysis of synaptosomal proteins in a model of IPS corroborated previously implicated mechanisms in neonatal $\mathrm{HI}$ brain injury and uncovered novel molecular networks implicated in synapse formation and neuro- transmission. This study adds to the body of work explaining the pathophysiology of mild to moderate HI brain injury during the neonatal period [74-76]. Moreover, the observation that IPS causes more neurological deficits in males, in both mice and humans [7-9], highlights that mouse models of IPS accurately reflect clinical IPS phenomena. As such, studies on mice can help elucidate the molecular pathways underlying the clinical manifestations of IPS. Finally, the findings underscore therapeutic approaches that aim to enhance synaptic plasticity as a treatment for the long-term neurological dysfunctions following IPS [77], and highlight novel signaling networks for potential combinatorial therapeutic strategies for IPS-associated neurodevelopmental effects.

\section{Acknowledgments}

We would like to thank Todd Markowski and LeeAnn Higgins of Center for Mass Spectrometry and Proteomics for their generous assistance with the iTRAQ analysis, and Cynthia Zheng for assisting with surgical procedures and post-op care.

\section{Statement of Ethics}

The University of Minnesota Institutional Animal Care and Use Committee approved all experiments in this study according to the Ethical and Responsible Animal Care in Research Guidelines.

\section{Disclosure Statement}

The authors declare that there are no conflicts or financial interests.

\section{Funding Sources}

The Viking's Children Fund, Masonic Children Hospital, and Minnesota Medical Foundation provided funding to PVT.

\section{Author Contributions}

A.K.B. performed ischemic surgery, collected synaptosomes, and prepared the manuscript. M.M.B. assisted in ischemic surgery, provided postsurgical care, and collected body temperature and behavioral data. T.M. performed and analyzed behavioral data. J.J.G. isolated proteins and performed Western blot analysis. R.R. provided intellectual discussion. P.V.T. conceptualized and supervised the study, performed surgery, prepared the manuscript, and had the primary responsibility for the final content. All authors reviewed and approved the final draft. 


\section{References}

1 Raju TN, Nelson KB, Ferriero D, Lynch JK; NICHD-NINDS Perinatal Stroke Workshop Participants. Ischemic perinatal stroke: summary of a workshop sponsored by the National Institute of Child Health and Human Development and the National Institute of Neurological Disorders and Stroke. Pediatrics. 2007 Sep;120(3):609-16.

2 Fernández-López D, Natarajan N, Ashwal S, Vexler ZS. Mechanisms of perinatal arterial ischemic stroke. J Cereb Blood Flow Metab. 2014 Jun;34(6):921-32.

3 Lehman LL, Rivkin MJ. Perinatal arterial ischemic stroke: presentation, risk factors, evaluation, and outcome. Pediatr Neurol. 2014 Dec; 51(6):760-8.

4 Tsze DS, Valente JH. Pediatric stroke: a review. Emerg Med Int. 2011;2011:734506.

5 Raju TN. Ischemic perinatal stroke: challenge and opportunities. Int J Stroke. 2008;3:169-72.

6 Kirton A, Deveber G. Life after perinatal stroke. Stroke. 2013 Nov;44(11):3265-71.

7 Charriaut-Marlangue C, Besson VC, Baud O. Sexually Dimorphic Outcomes after Neonatal Stroke and Hypoxia-Ischemia. Int J Mol Sci. 2017 Dec;19(1):19.

8 Smith AL, Alexander M, Rosenkrantz TS, Sadek ML, Fitch RH. Sex differences in behavioral outcome following neonatal hypoxia ischemia: insights from a clinical meta-analysis and a rodent model of induced hypoxic ischemic brain injury. Exp Neurol. 2014 Apr;254:54-67.

9 Golomb MR, Fullerton HJ, Nowak-Gottl U, Deveber G; International Pediatric Stroke Study Group. Male predominance in childhood ischemic stroke: findings from the international pediatric stroke study. Stroke. 2009 Jan;40(1):52-7.

10 Johnston MV. Excitotoxicity in perinatal brain injury. Brain Pathol. 2005 Jul;15(3):234-40.

11 Ten VS, Wu EX, Tang H, Bradley-Moore M, Fedarau MV, Ratner VI, \$. Late measures of brain injury after neonatal hypoxia-ischemia in mice. Stroke. 2004 Sep;35(9):2183-8.

12 Wiersma AM, Fouad K, Winship IR. Enhancing Spinal Plasticity Amplifies the Benefits of Rehabilitative Training and Improves Recovery from Stroke. J Neurosci. 2017 Nov;37(45): 10983-97.

13 Seghier ML, Lazeyras F, Zimine S, Maier SE, Hanquinet S, Delavelle J, et al. Combination of event-related fMRI and diffusion tensor imaging in an infant with perinatal stroke. Neuroimage. 2004 Jan;21(1):463-72.

14 Costain WJ, Rasquinha I, Sandhu JK, Rippstein P, Zurakowski B, Slinn J, et al. Cerebral ischemia causes dysregulation of synaptic adhesion in mouse synaptosomes. J Cereb Blood Flow Metab. 2008 Jan;28(1):99-110.

15 Mariucci G, Tantucci M, Giuditta A, Ambrosini MV. Permanent brain ischemia induces marked increments in hsp72 expression and local protein synthesis in synapses of the ischemic hemisphere. Neurosci Lett. 2007 Mar; 415(1):77-80.
16 Weinberger J, Cohen G. The differential effect of ischemia on the active uptake of dopamine, gamma-aminobutyric acid, and glutamate by brain synaptosomes. J Neurochem. 1982 Apr; 38(4):963-8.

17 Merali Z, Gao MM, Bowes T, Chen J, Evans K, Kassner A. Neuroproteome changes after ischemia/reperfusion injury and tissue plasminogen activator administration in rats: a quantitative iTRAQ proteomics study. PLoS One. 2014 May;9(5):e98706.

18 Shao G, Wang Y, Guan S, Burlingame AL, Lu F, Knox R, et al. Proteomic Analysis of Mouse Cortex Postsynaptic Density following Neonatal Brain Hypoxia-Ischemia. Dev Neurosci. 2017;39(1-4):66-81.

19 He Q, Man L, Ji Y, Zhang S, Jiang M, Ding F, et al. Comparative proteomic analysis of differentially expressed proteins between peripheral sensory and motor nerves. J Proteome Res. 2012 Jun;11(6):3077-89.

20 Moczulska KE, Pichler P, Schutzbier M, Schleiffer A, Rumpel S, Mechtler K. Deep and precise quantification of the mouse synaptosomal proteome reveals substantial remodeling during postnatal maturation. J Proteome Res. 2014 Oct;13(10):4310-24.

21 Tran PV, Dakoji S, Reise KH, Storey KK, Georgieff MK. Fetal iron deficiency alters the proteome of adult rat hippocampal synaptosomes. Am J Physiol Regul Integr Comp Physiol. 2013 Dec;305(11):R1297-306.

22 Semple BD, Blomgren K, Gimlin K, Ferriero DM, Noble-Haeusslein LJ. Brain development in rodents and humans: identifying benchmarks of maturation and vulnerability to injury across species. Prog Neurobiol. 2013 JulAug;106-107:1-16.

23 Albéri L, Chi Z, Kadam SD, Mulholland JD, Dawson VL, Gaiano N, et al. Neonatal stroke in mice causes long-term changes in neuronal Notch-2 expression that may contribute to prolonged injury. Stroke. 2010 Oct;41(10 Suppl):S64-71.

24 Felt BT, Schallert T, Shao J, Liu Y, Li X, Barks JD. Early appearance of functional deficits after neonatal excitotoxic and hypoxic-ischemic injury: fragile recovery after development and role of the NMDA receptor. Dev Neurosci. 2002;24(5):418-25.

25 Isayama K, Pitts LH, Nishimura MC. Evaluation of 2,3,5-triphenyltetrazolium chloride staining to delineate rat brain infarcts. Stroke. 1991 Nov;22(11):1394-8.

26 Carlin RK, Grab DJ, Cohen RS, Siekevitz P. Isolation and characterization of postsynaptic densities from various brain regions: enrichment of different types of postsynaptic densities. J Cell Biol. 1980 Sep;86(3):831-45.

27 Lin WY, Chang YC, Lee HT, Huang CC. CREB activation in the rapid, intermediate, and delayed ischemic preconditioning against hypoxic-ischemia in neonatal rat. J Neurochem. 2009 Feb;108(4):847-59.
28 Wang X, Zhu C, Qiu L, Hagberg H, Sandberg M, Blomgren K. Activation of ERK1/2 after neonatal rat cerebral hypoxia-ischaemia. J Neurochem. 2003 Jul;86(2):351-62.

29 Buser JR, Maire J, Riddle A, Gong X, Nguyen T, Nelson K, et al. Arrested preoligodendrocyte maturation contributes to myelination failure in premature infants. Ann Neurol. 2012 Jan;71(1):93-109.

30 Segovia KN, McClure M, Moravec M, Luo NL, Wan Y, Gong X, et al. Arrested oligodendrocyte lineage maturation in chronic perinatal white matter injury. Ann Neurol. 2008 Apr; 63(4):520-30.

31 Faustino JV, Wang X, Johnson CE, Klibanov A, Derugin N, Wendland MF, et al. Microglial cells contribute to endogenous brain defenses after acute neonatal focal stroke. J Neurosci. 2011 Sep;31(36):12992-3001.

32 Northington FJ, Chavez-Valdez R, Martin LJ. Neuronal cell death in neonatal hypoxia-ischemia. Ann Neurol. 2011 May;69(5):743-58.

33 Walton M, Connor B, Lawlor P, Young D, Sirimanne E, Gluckman P, et al. Neuronal death and survival in two models of hypoxic-ischemic brain damage. Brain Res Brain Res Rev. 1999 Apr;29(2-3):137-68.

34 Gonzalez FF, Larpthaveesarp A, McQuillen P, Derugin N, Wendland M, Spadafora R, et al. Erythropoietin increases neurogenesis and oligodendrogliosis of subventricular zone precursor cells after neonatal stroke. Stroke. 2013 Mar;44(3):753-8.

35 Spadafora R, Gonzalez FF, Derugin N, Wendland M, Ferriero D, McQuillen P. Altered fate of subventricular zone progenitor cells and reduced neurogenesis following neonatal stroke. Dev Neurosci. 2010 Jul;32(2):101-13.

36 Kadam SD, Mulholland JD, McDonald JW, Comi AM. Neurogenesis and neuronal commitment following ischemia in a new mouse model for neonatal stroke. Brain Res. 2008 May;1208:35-45.

37 Asaithambi A, Ay M, Jin H, Gosh A, Anantharam V, Kanthasamy A, et al. Protein kinase D1 (PKD1) phosphorylation promotes dopaminergic neuronal survival during 6-OHDAinduced oxidative stress. PLoS One. 2014 May; 9(5):e96947.

38 Knox R, Zhao C, Miguel-Perez D, Wang S, Yuan J, Ferriero D, et al. Enhanced NMDA receptor tyrosine phosphorylation and increased brain injury following neonatal hypoxia-ischemia in mice with neuronal Fyn overexpression. Neurobiol Dis. 2013 Mar;51:113-9.

39 Stetler RA, Gao Y, Zhang L, Weng Z, Zhang F, $\mathrm{Hu}$ X, et al. Phosphorylation of HSP27 by protein kinase $\mathrm{D}$ is essential for mediating neuroprotection against ischemic neuronal injury. J Neurosci. 2012 Feb;32(8):2667-82.

40 Kim GS, Cho S, Nelson JW, Zipfel GJ, Han BH. TrkB agonist antibody pretreatment enhances neuronal survival and long-term sensory motor function following hypoxic ischemic injury in neonatal rats. PLoS One. 2014 Feb;9(2):e88962. 
41 Knox R, Brennan-Minnella AM, Lu F, Yang D, Nakazawa T, Yamamoto T, et al. NR2B phosphorylation at tyrosine 1472 contributes to brain injury in a rodent model of neonatal hypoxia-ischemia. Stroke. 2014 Oct;45(10): 3040-7.

42 Nijboer CH, Bonestroo HJ, Zijlstra J, Kavelaars A, Heijnen CJ. Mitochondrial JNK phosphorylation as a novel therapeutic target to inhibit neuroinflammation and apoptosis after neonatal ischemic brain damage. Neurobiol Dis. 2013 Jun;54:432-44.

43 Xie R, Cheng M, Li M, Xiong X, Daadi M, Sapolsky RM, et al. Akt isoforms differentially protect against stroke-induced neuronal injury by regulating mTOR activities. J Cereb Blood Flow Metab. 2013 Dec;33(12):1875-85.

44 Terasaki Y, Sasaki T, Yagita Y, Okazaki S, Sugiyama $\mathrm{Y}$, Oyama N, et al. Activation of NR2A receptors induces ischemic tolerance through CREB signaling. J Cereb Blood Flow Metab. 2010 Aug;30(8):1441-9.

45 Bell MT, Puskas F, Bennett DT, Herson PS, Quillinan N, Fullerton DA, et al. Dexmedetomidine, an $\alpha$-2a adrenergic agonist, promotes ischemic tolerance in a murine model of spinal cord ischemia-reperfusion. J Thorac Cardiovasc Surg. 2014 Jan;147(1):500-6.

46 Han BH, D'Costa A, Back SA, Parsadanian M, Patel S, Shah AR, et al. BDNF blocks caspase-3 activation in neonatal hypoxia-ischemia. Neurobiol Dis. 2000 Feb;7(1):38-53.

47 Carloni S, Girelli S, Buonocore G, Longini M, Balduini W. Simvastatin acutely reduces ischemic brain damage in the immature rat via Akt and CREB activation. Exp Neurol. 2009 Nov; 220(1):82-9.

48 Zhang H, Liu Y, Guan S, Qu D, Wang L, Wang $\mathrm{X}$, et al. An Orally Active Allosteric GLP-1 Receptor Agonist Is Neuroprotective in Cellular and Rodent Models of Stroke. PLoS One. 2016 Feb;11(2):e0148827.

49 Lombardi MS, van den Tweel E, Kavelaars A, Groenendaal F, van Bel F, Heijnen CJ. Hypoxia/ischemia modulates $\mathrm{G}$ protein-coupled receptor kinase 2 and beta-arrestin- 1 levels in the neonatal rat brain. Stroke. 2004 Apr;35(4): 981-6.

50 Ma D, Hossain M, Rajakumaraswamy N, Arshad M, Sanders RD, Franks NP, et al. Dexmedetomidine produces its neuroprotective effect via the alpha $2 \mathrm{~A}$-adrenoceptor subtype. Eur J Pharmacol. 2004 Oct;502(1-2):87-97.

51 Quinlan EM, Halpain S. Emergence of activitydependent, bidirectional control of microtubule-associated protein MAP2 phosphorylation during postnatal development. J Neurosci. 1996 Dec;16(23):7627-37.

52 Roberts LA, Large CH, Higgins MJ, Stone TW, O'Shaughnessy CT, Morris BJ. Increased expression of dendritic mRNA following the induction of long-term potentiation. Brain Res Mol Brain Res. 1998 May;56(1-2):38-44.
53 Hagberg H, Mallard C, Ferriero DM, Vannucci SJ, Levison SW, Vexler ZS, et al. The role of inflammation in perinatal brain injury. Nat Rev Neurol. 2015 Apr;11(4):192-208.

54 Wang S, Wu EX, Tam CN, Lau HF, Cheung PT, Khong PL. Characterization of white matter injury in a hypoxic-ischemic neonatal rat model by diffusion tensor MRI. Stroke. 2008 Aug;39(8):2348-53.

55 Ashwal S, Tone B, Tian HR, Chong S, Obenaus A. Comparison of two neonatal ischemic injury models using magnetic resonance imaging. Pediatr Res. 2007 Jan;61(1):9-14.

56 Cheng J, Liu J, Li X, Peng J, Han S, Zhang R, et al. Insulin-like growth factor-1 receptor polymorphism and ischemic stroke: a case-control study in Chinese population. Acta Neurol Scand. 2008 Nov; 118(5):333-8.

57 Zou W, Fang C, Ji X, Liang X, Liu Y, Han C, et al. Estrogen Receptor (ER)-a36 Is Involved in Estrogen- and Tamoxifen-Induced Neuroprotective Effects in Ischemic Stroke Models. PLoS One. 2015 Oct;10(10):e0140660.

58 Li D, Wang C, Yao Y, Chen L, Liu G, Zhang R, et al. mTORC1 pathway disruption ameliorates brain inflammation following stroke via a shift in microglia phenotype from M1 type to M2 type. FASEB J. 2016 Oct;30(10):3388-99.

59 Wei H, Li Y, Han S, Liu S, Zhang N, Zhao L, et al. $\mathrm{CPKC} \gamma$-Modulated Autophagy in Neurons Alleviates Ischemic Injury in Brain of Mice with Ischemic Stroke Through Akt-mTOR Pathway. Transl Stroke Res. 2016 Dec;7(6): 497-511.

60 Johnston MV, Hagberg H. Sex and the pathogenesis of cerebral palsy. Dev Med Child Neurol. 2007 Jan;49(1):74-8.

61 Nuñez J. Sex and steroid hormones in early brain injury. Rev Endocr Metab Disord. 2012 Sep;13(3):173-86

62 Serra C, Federici M, Buongiorno A, Senni MI, Morelli S, Segratella E, et al. Transgenic mice with dominant negative PKC-theta in skeletal muscle: a new model of insulin resistance and obesity. J Cell Physiol. 2003 Jul;196(1):89-97.

63 Yoshimoto T, Kanakaraj P, Ying Ma J, Cheng M, Kerr I, Malaiyandi L, et al. NXY-059 maintains Akt activation and inhibits release of cytochrome $\mathrm{C}$ after focal cerebral ischemia. Brain Res. 2002 Aug;947(2):191-8.

64 Doeppner TR, Doehring M, Bretschneider E, Zechariah A, Kaltwasser B, Müller B, et al. MicroRNA-124 protects against focal cerebral ischemia via mechanisms involving Usp14-dependent REST degradation. Acta Neuropathol. 2013 Aug;126(2):251-65.

65 Formisano L, Guida N, Valsecchi V, Cantile M, Cuomo O, Vinciguerra A, et al. Sp3/REST/ HDAC1/HDAC2 Complex Represses and Sp1/HIF-1/p300 Complex Activates ncx 1 Gene Transcription, in Brain Ischemia and in Ischemic Brain Preconditioning, by Epigenetic Mechanism. J Neurosci. 2015 May;35(19): 7332-48.
66 Mehta SL, Kim T, Vemuganti R. Long Noncoding RNA FosDT Promotes Ischemic Brain Injury by Interacting with REST-Associated Chromatin-Modifying Proteins. J Neurosci. 2015 Dec;35(50):16443-9.

67 Medhi B, Aggarwal R, Chakrabarti A. Neuroprotective effect of pioglitazone on acute phase changes induced by partial global cerebral ischemia in mice. Indian J Exp Biol. 2010 Aug; 48(8):793-9.

68 Zhao Y, Patzer A, Gohlke P, Herdegen T, Culman J. The intracerebral application of the PPARgamma-ligand pioglitazone confers neuroprotection against focal ischaemia in the rat brain. Eur J Neurosci. 2005 Jul;22(1):27882.

69 Luo Y, Yin W, Signore AP, Zhang F, Hong Z, Wang $S$, et al. Neuroprotection against focal ischemic brain injury by the peroxisome proliferator-activated receptor-gamma agonist rosiglitazone. J Neurochem. 2006 Apr;97(2): 435-48.

70 Ceprián M, Jiménez-Sánchez L, Vargas C, Barata L, Hind W, Martínez-Orgado J. Cannabidiol reduces brain damage and improves functional recovery in a neonatal rat model of arterial ischemic stroke. Neuropharmacology. 2017 Apr;116:151-9.

71 D’Angelo B, Ek CJ, Sun Y, Zhu C, Sandberg M, Mallard C. GSK3 $\beta$ inhibition protects the immature brain from hypoxic-ischaemic insult via reduced STAT3 signalling. Neuropharmacology. 2016 Feb;101:13-23.

72 Li L, McBride DW, Doycheva D, Dixon BJ, Krafft PR, Zhang JH, et al. G-CSF attenuates neuroinflammation and stabilizes the bloodbrain barrier via the PI3K/Akt/GSK-3 $\beta$ signaling pathway following neonatal hypoxia-ischemia in rats. Exp Neurol. 2015 Oct;272:13544.

73 Wang Z, Guan W, Han Y, Ren H, Tang X, Zhang $\mathrm{H}$, et al. Stimulation of Dopamine D3 Receptor Attenuates Renal Ischemia-Reperfusion Injury via Increased Linkage With Ga12. Transplantation. 2015 Nov;99(11):2274-84.

74 Bennet L, Van Den Heuij L, Dean JM, Drury P, Wassink G, Gunn AJ. Neural plasticity and the Kennard principle: does it work for the preterm brain? Clin Exp Pharmacol Physiol. 2013 Nov;40(11):774-84.

75 Curristin SM, Cao A, Stewart WB, Zhang H, Madri JA, Morrow JS, et al. Disrupted synaptic development in the hypoxic newborn brain Proc Natl Acad Sci USA. 2002 Nov;99(24): 15729-34.

76 Dean JM, McClendon E, Hansen K, Azimi-Zonooz A, Chen K, Riddle A, et al. Prenatal cerebral ischemia disrupts MRI-defined cortical microstructure through disturbances in neuronal arborization. Sci Transl Med. 2013 Jan; 5(168):168ra7.

77 Johnston MV. Plasticity in the developing brain: implications for rehabilitation. Dev Disabil Res Rev. 2009;15(2):94-101. 